\title{
SOLVABILITY OF SEMILINEAR EQUATIONS WITH COMPACT PERTURBATIONS OF OPERATORS OF MONOTONE TYPE
}

\author{
ZHENGYUAN GUAN
}

(Communicated by Palle E. T. Jorgensen)

\begin{abstract}
The solvability of the equation $A u-T u+C u=f$ is studied under various assumptions of monotonicity and compactness on the operators $A, T$, and $C$, which map subsets of a reflexive Banach space $X$ into its dual space. It is nowhere assumed that $X$ possesses a Schauder basis or that the operator $T$ is positive definite and selfadjoint. The results extend and/or improve recent results obtained by Chen, Kartsatos and Mabry, and Kesavan.
\end{abstract}

\section{INTRODUCTION AND PRELIMINARIES}

Let $X$ be a real reflexive Banach space with dual space $X^{*}$. By Trojanski's Theorem (see Pascali and Sburlan [12]), there exist equivalent norms on $X$ and $X^{*}$ such that both spaces, which are still dual to each other, are locally uniformly convex. From this point on we assume that $X$ is a reflexive Banach space with both $X$ and $X^{*}$ locally uniformly convex. For any $x \in X, y \in X^{*}$, we denote $y(x)$ by $(y, x)$. If $\left\{x_{n}\right\}$ is a sequence in $X, x_{n} \rightarrow x_{0}$ means that $x_{n}$ converges strongly to $x_{0}$ and $x_{n} \rightarrow x_{0}$ means that $x_{n}$ converges weakly to $x_{0}$.

A mapping $T$ which maps $D(T) \subset X$ into $X^{*}$ is said to be compact if $T$ is continuous and maps bounded sets of $X$ into relatively compact sets of $X^{*}$. The mapping $T$ is said to be completely continuous if $x_{n}-x_{0}$ implies that $T x_{n} \rightarrow T x_{0}$. It is well known that, for a linear mapping, complete continuity and compactness are equivalent and a completely continuous mapping with closed convex domain is compact.

A mapping $T: D(T) \subset X \rightarrow X^{*}$ is said to be monotone if for any $x, y \in$ $D(T)$ we have $(T x-T y, x-y) \geq 0$; strictly monotone if $(T x-T y, x-y)>0$ for any $x \neq y$; and strongly monotone if $(T x-T y, x-y) \geq m\|x-y\|^{2}$ for some positive constant $m$ and any $x, y \in D(T)$.

The duality mapping $J: X \rightarrow 2^{X^{*}}$ is defined by

$$
J(x)=\left\{x^{*} \in X^{*}:\left(x^{*}, x\right)=\|x\|^{2}=\left\|x^{*}\right\|^{2}\right\}
$$

Received by the editors April 27, 1992 and, in revised form, August 3, 1992.

1991 Mathematics Subject Classification. Primary 47H15, 47H17, 47H05.

Key words and phrases. Semilinear equations, monotone operators, compact perturbations.

This paper is based in part on the author's Ph.D. dissertation research at the University of South Florida under the guidance of Dr. A. G. Kartsatos. 
for any $x \in X$. By the Hahn-Banach Theorem, $J(x)$ is nonempty for any $x$ in $X$. In our setting the duality mapping $J$ is single valued, bicontinuous, strictly monotone and such that $J(\lambda x)=\lambda J(x)$ for any $\lambda \in R, x \in X$ (see Browder [2]).

A mapping $T: D(T) \subset X \rightarrow X^{*}$ is said to be maximal monotone if $T$ is monotone and $R(T+\lambda J)=X^{*}$ for any $\lambda>0$. This definition is equivalent to the following: $T$ is monotone and $\left(T x-y_{0}, x-x_{0}\right) \geq 0$ for any $x \in D(T)$ implies $x_{0} \in D(T)$ and $T x_{0}=y_{0}$. We denote by $B(0, b)$ the open ball in $X$ or $X^{*}$ with center at 0 and radius $b>0$. The symbols $\bar{B}$ and $\partial B$ stand for the closure and the boundary of the set $B$, respectively. We use the letters $R$ and $R_{+}$to denote the real line and the set $[0, \infty)$, respectively.

Kesavan [8] established the solvability of equation

$$
u-T u+C u=f
$$

for $T$ and $C$ mapping real Hilbert space $H$ into $H$ under the following conditions: $T$ is a continuous linear operator which is also symmetric, i.e., $(T u, v)=(u, T v)$ for all $u, v \in H$, positive definite, i.e., $(T v, v)>0$ for all $v \in H, v \neq 0$, and $T$ is compact; $C$ is completely continuous, which satisfies $(C u, u) \geq 0$ for all $u \in H$, and $(C u, u)=0$ implies $u=0$; and there exists $p>1$ such that for all $r \geq 0$ and for all $u \in H, C(r u)=r^{p} C(u)$.

Kesavan used the Galerkin method to prove the above result. Under the assumptions that $T$ is symmetric, positive definite, and compact, there exists a sequence of eigenvalues of $T,\left\{\lambda_{j}\right\}$, which decreases to zero, and a corresponding sequence of eigenvectors $\left\{w_{j}\right\}$, which forms an orthonormal basis for $H$. Let $V_{m}$ be the space spanned by the eigenvectors $\left\{w_{1}, w_{2}, \ldots, w_{m}\right\}$. The finite-dimensional problem of finding $u_{m} \in V_{m}$ such that

$$
\left(u_{m}, v\right)-\left(T u_{m}, v\right)+\left(C u_{m}, v\right)=(f, v)
$$

for all $v \in V_{m}$ was considered in [8] for any $m \in Z^{+}$. The solvability of (*) and the uniform boundedness of the solutions $\left\{u_{m}\right\}$ were established. Then, by the complete continuity of $C$ and $T$, the solvability of (1) was obtained.

Kartsatos and Mabry [7] considered the equation

$$
A u-T u+C u=f
$$

for $A: D(A) \subset H \rightarrow H$ maximal monotone and strongly monotone. Kesavan's result was extended, and localized versions of it were also obtained. These authors used degree theory and the main idea in Kesavan's argument. Their results were recently extended to Banach spaces by Chen [4]. Chen considered the problem, which was posed by Kartsatos and Mabry [7], in reflexive Banach spaces possessing a Schauder basis.

The main difficulty in obtaining the solvability of (1) and (2) is due to the fact that the operator $C$, which satisfies the conditions mentioned above, is not global coercive, i.e., it is impossible to have $(C u, u) \geq k\|u\|^{p+1}$ for all $u \in H$ and some $k>0$. My contribution in this paper lies mainly in the observation that even if $C$ is not global coercive, we can obtain $(C u, u) \geq k\|u\|^{p+1}$ for the solutions $u$ of (1) or (2) and some constant $k>0$, as you will see in the proofs of my results. Following from that observation, the solvability of the equation is established by an approach that is different from what was employed in $[4,7$, 8]. With the new approach, the assumption that $T$ is symmetric and positive 
definite becomes unnecessary. Also as a result of the new approach the existence of an orthonormal basis for the underlying space, which is crucial in $[4,7,8]$, becomes obsolete; the extension of the problem from separable Hilbert spaces or Banach spaces with Schauder basis to reflexive Banach spaces is obtained. Thus, our results are significant generalizations and improvements of various results in $[4,7,8]$.

\section{MAIN RESULTS}

Theorem 1. Let $X$ be a reflexive Banach space. Let $A: D(A) \subset X \rightarrow X^{*}$ be maximal monotone and strongly monotone with $0 \in D(A)$, and let $A(0)=0$. Let $T: X \rightarrow X^{*}$ be linear and compact and $C: X \rightarrow X^{*}$ completely continuous. Assume further that there exists a constant $p>1$ and a completely continuous function $g: \overline{B(0,1)} \rightarrow R_{+}$such that $g(u)=0$ implies $u=0$ and $(C u, u) \geq$ $g(u /\|u\|)\|u\|^{p+1}$ for all $u \in X$ with $u \neq 0$. Then equation (2) has at least one solution for any $f \in X^{*}$.

Proof. We consider the equation

$$
A u+\frac{1}{m} J u-T u+C u-f=0
$$

for any positive integer $m$.

Since $A$ is maximal monotone, we can define $R_{m}=\left(A+\frac{1}{m} J\right)^{-1}: X^{*} \rightarrow$ $D(A) . A$ is strongly monotone; it is easy to show that $R_{m}$ is continuous. Now, (3) is equivalent to

$$
u-R_{m}(T u-C u+f)=0 .
$$

Since $R_{m}$ is continuous and $C$ and $T$ are compact, $R_{m}(T-C+f): X \rightarrow$ $D(A)$ is compact. By the Leray-Schauder degree theory (see Lloyd [9]), (4) is solvable if we can show that there exists $b>0$ such that $u-t R_{m}(T u-C u+f) \neq$ 0 for any $t \in(0,1], u \in \partial B(0, b)$. Equivalently, we only need to show that the solutions of $u-t R_{m}(T u-C u+f)=0$ for any $t \in(0,1]$ are uniformly bounded.

To this end, we first show that there exists $\varepsilon>0$ such that $g\left(u_{t} /\left\|u_{t}\right\|\right)>\varepsilon$ for any solution $u_{t}$ of $u-t R_{m}(T u-C u+f)=0$ with $t \in(0,1],\left\|u_{t}\right\| \geq 1$.

If this is not true, there exists $\left\{t_{n}\right\} \subset(0,1]$ and $\left\{u_{n}\right\} \subset X,\left\|u_{n}\right\| \geq 1$, with

$$
u_{n}-t_{n} R_{m}\left(T u_{n}-C u_{n}+f\right)=0
$$

and $g\left(u_{n} /\left\|u_{n}\right\|\right) \rightarrow 0$.

Since $X$ is reflexive Banach space, $\overline{B(0,1)}$ is weakly compact. Thus, there exists a subsequence of $\left\{u_{n}\right\}$, say $\left\{u_{j}\right\}$, such that $u_{j} /\left\|u_{j}\right\| \rightarrow u_{0}$ for some $u_{0} \in \overline{B(0,1)}$. Since $g$ is completely continuous, $g\left(u_{j} /\left\|u_{j}\right\|\right) \rightarrow g\left(u_{0}\right)$. Thus, $g\left(u_{0}\right)=0$. By our assumption on $g$, we have $u_{0}=0$. Hence $u_{j} /\left\|u_{j}\right\| \rightarrow 0$.

Since $u_{j}-t_{j} R_{m}\left(T u_{j}-C u_{j}+f\right)=0$, we have

$$
A\left(\frac{u_{j}}{t_{j}}\right)+\frac{1}{m} J\left(\frac{u_{j}}{t_{j}}\right)-T u_{j}+C u_{j}-f=0 .
$$


Notice that $u_{j} / t_{j} \in D(A)$, since $R_{m}: X^{*} \rightarrow D(A)$. Thus,

$$
\begin{aligned}
\frac{1}{m} J u_{j} & =-t_{j} A\left(\frac{u_{j}}{t_{j}}\right)+t_{j} T u_{j}-t_{j} C u_{j}+t_{j} f \\
\left(\frac{1}{m} J u_{j}, u_{j}\right) & =-t_{j}\left(A\left(\frac{u_{j}}{t_{j}}\right), u_{j}\right)+t_{j}\left(T u_{j}, u_{j}\right)-t_{j}\left(C u_{j}, u_{j}\right)+t_{j}\left(f, u_{j}\right) \\
& \leq t_{j}\left(T u_{j}, u_{j}\right)+t_{j}\left(f, u_{j}\right),
\end{aligned}
$$

which implies

$$
\frac{1}{m}=\left(\frac{1}{m} J \frac{u_{j}}{\left\|u_{j}\right\|}, \frac{u_{j}}{\left\|u_{j}\right\|}\right) \leq t_{j}\left(T \frac{u_{j}}{\left\|u_{j}\right\|}, \frac{u_{j}}{\left\|u_{j}\right\|}\right)+t_{j}\left(f, \frac{u_{j}}{\left\|u_{j}\right\|}\right) \frac{1}{\left\|u_{j}\right\|} \rightarrow 0
$$

as $j \rightarrow \infty$, i.e., a contradiction. Hence, we have $g\left(u_{t} /\left\|u_{t}\right\|\right)>\varepsilon$ for some $\varepsilon>0$.

Now, if $u_{t}-t R_{m}\left(T u_{t}-C u_{t}+f\right)=0$ with $\left\|u_{t}\right\| \geq 1$, then

$$
A\left(\frac{u_{t}}{t}\right)+\frac{1}{m} J \frac{u_{t}}{t}-T u_{t}+C u_{t}-f=0
$$

and

$$
\begin{aligned}
0 & =\left(A\left(\frac{u_{t}}{t}\right), u_{t}\right)+\frac{1}{m}\left(J\left(\frac{u_{t}}{t}\right), u_{t}\right)-\left(T u_{t}, u_{t}\right)+\left(C u_{t}, u_{t}\right)-\left(f, u_{t}\right) \\
& \geq-\|T\|\left\|u_{t}\right\|^{2}+g\left(\frac{u_{t}}{\left\|u_{t}\right\|}\right)\left\|u_{t}\right\|^{p+1}-\|f\|\left\|u_{t}\right\| \\
& \geq-\|T\|\left\|u_{t}\right\|^{2}+\varepsilon\left\|u_{t}\right\|^{p+1}-\|f\|\left\|u_{t}\right\| .
\end{aligned}
$$

Since $p>1,\left\|u_{t}\right\|$ must be uniformly bounded. Hence, we get that (3) and (4) are solvable for any integer $m$.

Now we are going to show that the solutions of (3) are uniformly bounded with respect to $m$. If this is not true, we may assume that there exists $\left\{u_{n}\right\}$, $A u_{n}+\frac{1}{n} J u_{n}-T u_{n}+C u_{n}-f=0$, and $\left\|u_{n}\right\| \rightarrow \infty$. We may assume that $\left\|u_{n}\right\| \geq 1$.

First we show that there exists $\varepsilon>0$ such that $g\left(u_{n} /\left\|u_{n}\right\|\right)>\varepsilon$ for any $u_{n} \in\left\{u_{n}\right\}$.

If this is not true, there exists a subsequence of $\left\{u_{n}\right\}$, again called $\left\{u_{n}\right\}$, such that $g\left(u_{n} /\left\|u_{n}\right\|\right) \rightarrow 0$. Since $\overline{B(0,1)}$ is weakly compact, we may assume that $u_{n} /\left\|u_{n}\right\| \rightarrow u_{0}$ for some $u_{0} \in \overline{B(0,1)}$. Since $g$ is completely continuous, $g\left(u_{0}\right)=\lim _{n \rightarrow \infty} g\left(u_{n} /\left\|u_{n}\right\|\right)=0$. By our assumption on $g, u_{0}=0$, so we have $u_{n} /\left\|u_{n}\right\| \rightarrow 0$. Now,

$$
\begin{gathered}
T u_{n}=A u_{n}+\frac{1}{n} J u_{n}+C u_{n}-f, \\
\left(T u_{n}, u_{n}\right) \geq\left(A u_{n}, u_{n}\right)-\left(f, u_{n}\right) \geq m\left\|u_{n}\right\|^{2}-\left(f, u_{n}\right),
\end{gathered}
$$

where $m$ is the strong monotonicity constant of $A$. So

$$
\left(T \frac{u_{n}}{\left\|u_{n}\right\|}, \frac{u_{n}}{\left\|u_{n}\right\|}\right) \geq m-\frac{1}{\left\|u_{n}\right\|}\left(f, \frac{u_{n}}{\left\|u_{n}\right\|}\right) \rightarrow m>0 .
$$

Since $T$ is linear and compact, $T u_{n} /\left\|u_{n}\right\| \rightarrow 0$. Thus, $\left(T u_{n} /\left\|u_{n}\right\|, u_{n} /\left\|u_{n}\right\|\right) \rightarrow$ 0 , which is a contradiction, so we have $g\left(u_{n} /\left\|u_{n}\right\|\right)>\varepsilon$ for some $\varepsilon>0$. 
Now

$$
\begin{aligned}
0 & =\left(A u_{n}, u_{n}\right)+\left(\frac{1}{n} J u_{n}, u_{n}\right)-\left(T u_{n}, u_{n}\right)+\left(C u_{n}, u_{n}\right)-\left(f, u_{n}\right) \\
& \geq-\|T\|\left\|u_{n}\right\|^{2}+\varepsilon\left\|u_{n}\right\|^{p+1}-\|f\|\left\|u_{n}\right\| \rightarrow \infty
\end{aligned}
$$

since $p>1$, and $\left\|u_{n}\right\| \rightarrow \infty$. By this contradiction, we get that $\left\{u_{n}\right\}$ is uniformly bounded.

Now, since $\left\{u_{n}\right\}$ is bounded and $X$ is reflexive, we may assume that $u_{n} \rightarrow$ $u$, for some $u \in X$. Then $\frac{1}{n} u_{n} \rightarrow 0$ and by our assumptions, $C u_{n} \rightarrow C u$, $T u_{n} \rightarrow T u$. So, $A u_{n} \rightarrow T u-C u+f$. Since $A$ is monotone, we have $(A x-$ $\left.A u_{n}, x-u_{n}\right) \geq 0$ for any $x \in D(A)$, so $(A x-(T u-C u+f), x-u) \geq 0$. Since $A$ is maximal monotone, we have $u \in D(A)$, and $A u=T u-C u+f$ or $A u-T u+C u=f$.

Corollary 1. Let $H$ be a real Hilbert space. Let $T: H \rightarrow H$ be linear and compact and $C: H \rightarrow H$ completely continuous. Assume further that there exist $p>1$ and a completely continuous function $g: \overline{B(0,1)} \rightarrow R_{+}$such that $g(u)=0$ implies $u=0$ and $(C u, u) \geq g(u /\|u\|)\|u\|^{p+1}$ for all $u \in H$. Then, for any $f \in H$, (1) has at least one solution.

Corollary 1 is an improvement of Kesavan's result. In Corollary 1, we do not assume that $H$ is separable and $T$ is symmetric and positive definite. The assumptions on $C$ are also more general in Corollary 1 than the one in Kesavan's Theorem [8], but this is due to Kartsatos and Mabry [7].

A mapping $T: D(T) \subset X \rightarrow X^{*}$ is said to be demicontinuous if $T$ is continuous from the strong topology of $X$ to the weak topology of $X^{*} ; T$ is said to be of type $(S)$ if for any sequence $\left\{x_{n}\right\} \subset D(T)$ with $x_{n} \rightarrow x \in X$ and $\lim _{n \rightarrow \infty}\left(T x_{n}, x_{n}-x\right)=0$, we have $x_{n} \rightarrow x$, and $T$ is said to be of type $\left(S_{+}\right)$ if, for any $\left\{x_{n}\right\} \subset D(T)$ with $x_{n} \rightarrow x \in X$ and $\limsup _{n \rightarrow \infty}\left(T x_{n}, x_{n}-x\right) \leq 0$, we have $x_{n} \rightarrow x$.

As in Theorem 1, but without assuming that $A$ is strongly monotone, we have

Theorem 2. Let $A: X \rightarrow X^{*}$ be maximal monotone and of type $(S), A(0)=0$, and $(A r u, u) \leq r(A u, u)$ for any $u \in X, 0 \leq r \leq 1$. Let $T, C$ be as in Theorem 1. Then, for any $f \in X^{*}$, (2) has at least one solution.

Proof. As in the proof of Theorem 1, we can show that (3) is solvable for any integer $m$. Notice that the continuity of $R_{m}$ can be derived from the fact that $J$ is of type $\left(S_{+}\right)$.

Now we are going to show that the solutions of (3) are uniformly bounded with respect to $m$. If this is not true, we may assume that there exists $\left\{u_{n}\right\} \subset X$ such that $\left\|u_{n}\right\| \rightarrow \infty$ and $A u_{n}+\frac{1}{n} J u_{n} T u_{n}+C u_{n}-f=0$. We can assume that $\left\|u_{n}\right\| \geq 1$.

First we show $g\left(u_{n} /\left\|u_{n}\right\|\right) \geq \varepsilon$ for some $\varepsilon>0$ and any $n$.

If this is not true, there exists a subsequence of $\left\{u_{n}\right\}$, again called $\left\{u_{n}\right\}$, such that $g\left(u_{n} /\left\|u_{n}\right\|\right) \rightarrow 0$. Since $X$ is a reflexive Banach space, we can assume that $u_{n} /\left\|u_{n}\right\| \rightarrow u_{0}$ for some $u_{0}$ in $\overline{B(0,1)}$. Since $g$ is completely continuous, $g\left(u_{0}\right)=\lim _{n \rightarrow \infty} g\left(u_{n} /\left\|u_{n}\right\|\right)=0$. By our assumption on $g, u_{0}=0$. We have 
$u_{n} /\left\|u_{n}\right\| \rightarrow 0$. Now, $A u_{n}+\frac{1}{n} J u_{n}-T u_{n}+C u_{n}-f=0$, and

$$
\begin{aligned}
&\left(A u_{n}, u_{n}\right)=-\frac{1}{n}\left(J u_{n}, u_{n}\right)-\left(C u_{n}, u_{n}\right)+\left(T u_{n}, u_{n}\right)+\left(f, u_{n}\right) \\
& \leq\left(T u_{n}, u_{n}\right)+\left(f, u_{n}\right) \\
& \frac{1}{\left\|u_{n}\right\|}\left(A u_{n}, \frac{u_{n}}{\left\|u_{n}\right\|}\right) \leq\left(T \frac{u_{n}}{\left\|u_{n}\right\|}, \frac{u_{n}}{\left\|u_{n}\right\|}\right)+\frac{1}{\left\|u_{n}\right\|}\left(f, \frac{u_{n}}{\left\|u_{n}\right\|}\right) .
\end{aligned}
$$

By our assumption on $A$, since $\left\|u_{n}\right\| \geq 1$, we have

$$
\left(A \frac{u_{n}}{\left\|u_{n}\right\|}, \frac{u_{n}}{\left\|u_{n}\right\|}\right) \leq\left(T \frac{u_{n}}{\left\|u_{n}\right\|}, \frac{u_{n}}{\left\|u_{n}\right\|}\right)+\frac{1}{\left\|u_{n}\right\|}\left(f, \frac{u_{n}}{\left\|u_{n}\right\|}\right) .
$$

Since $u_{n} /\left\|u_{n}\right\| \rightarrow 0,\left\|u_{n}\right\| \rightarrow \infty$, and $T$ is linear and compact (this implies $T$ is completely continuous), we have $\lim _{\sup _{n \rightarrow \infty}}\left(A \frac{u_{n}}{\left\|u_{n}\right\|}, \frac{u_{n}}{\left\|u_{n}\right\|}\right) \leq 0$, but since $A$ is monotone and $A(0)=0$,

$$
\left(A \frac{u_{n}}{\left\|u_{n}\right\|}, \frac{u_{n}}{\left\|u_{n}\right\|}\right)=\left(A \frac{u_{n}}{\left\|u_{n}\right\|}-A(0), \frac{u_{n}}{\left\|u_{n}\right\|}-0\right) \geq 0 .
$$

So $\lim _{n \rightarrow \infty}\left(A u_{n} /\left\|u_{n}\right\|, u_{n} /\left\|u_{n}\right\|\right)=0$. Now, since $A$ is of type $(S)$, we have $u_{n} /\left\|u_{n}\right\| \rightarrow 0$. This is a contradiction, so we have proved that $g\left(u_{n} /\left\|u_{n}\right\|\right) \geq \varepsilon$ for some $\varepsilon>0$.

Thus,

$$
\begin{aligned}
0 & =\left(A u_{n}, u_{n}\right)+\frac{1}{n}\left(J u_{n}, u_{n}\right)-\left(T u_{n}, u_{n}\right)+\left(C u_{n}, u_{n}\right)-\left(f, u_{n}\right) \\
& \geq-\|T\|\left\|u_{n}\right\|^{2}+\varepsilon\left\|u_{n}\right\|^{p+1}-\|f\|\left\|u_{n}\right\| \rightarrow \infty
\end{aligned}
$$

since $p>1$, and $\left\|u_{n}\right\| \rightarrow \infty$. By this contradiction, we get that $\left\{u_{n}\right\}$ is uniformly bounded.

The rest of the proof follows as in the proof of Theorem 1.

If $A: X \rightarrow X^{*}$ is demicontinuous and strongly monotone, then $A$ is maximal monotone and of type $(S)$. In fact, if $A$ is demicontinuous and satisfies $(A x-$ $A y, x-y) \geq \Phi(\|x-y\|)$ for any $x, y \in X$, where $\Phi: R_{+} \rightarrow R_{+}$is strictly increasing and continuous in a neighborhood of zero and such that $\Phi(0)=0$, then $A$ is also maximal monotone and of type $(S)$. If $A(r u)=r^{q} A u$ for some $q \geq 1$ and all $r \in[0,1]$, then $(A r u, u) \leq r(A u, u)$ for the same $r$.

A mapping $T: D(T) \subset X \rightarrow X^{*}$ is said to be quasimonotone if for any sequence $\left\{x_{n}\right\} \subset D(T)$ such that $x_{n} \rightarrow x \in X$, we have

$$
\limsup _{n \rightarrow \infty}\left(T x_{n}, x_{n}-x\right) \geq 0 \text {; }
$$

$T$ is said to be of type $(M)$ if, for any sequence $\left\{x_{n}\right\} \subset D(T)$ with $x_{n} \rightarrow x \in$ $D(T), T x_{n}-y$, and $\limsup _{n \rightarrow \infty}\left(T x_{n}, x_{n}-x\right) \leq 0$, we have $y=T x$; and $T$ is said to be generalized pseudomonotone if, for any sequence $\left\{x_{n}\right\} \subset D(T)$ with $x_{n} \rightarrow x \in D(T), T x_{n} \rightarrow y$, and $\lim _{\sup _{n \rightarrow \infty}}\left(T x_{n}, x_{n}-x\right) \leq 0$, we have $y=T x$ and $\left(T x_{n}, x_{n}\right) \rightarrow(T x, x)$.

Milojevic $[10,11]$ considered the solvability of (1) and (2) for $C$ or $A+C$ to be of type $(M)$ operator; the following theorem is an attempt in that direction. Further results will be presented in another paper of the author (cf. also Guan [6]). 
Theorem 3. Let $A, T, C: X \rightarrow X^{*}$ satisfy the following.

(1) $T$ is linear and compact;

(2) $C$ is demicontinuous, quasimonotone, and of type $(M)$; there exists $p>1$ such that $C(r u)=r^{p} C(u)$ for any $r \geq 0, u \in X ;(C u, u) \geq 0$ and $C(u)=0$ implies $u=0$;

(3) $A$ is demicontinuous and strongly monotone; there exists $q \in(0, p)$ such that $A(r u)=r^{q} A(u)$ for any $r \geq 0$ and $u \in X$; or

(4) $A$ is demicontinuous, monotone, and of type $(S)$; there exists $q \in[1, p)$ such that $A(r u)=r^{q} A(u)$ for any $r \geq 0$ and $u \in X$.

Then, for any $f \in X^{*}$, (2) has at least one solution.

Proof. For any fixed $f \in X^{*}$, we consider $S: X \rightarrow X^{*}, S u=A u-T u+C u-f$, $u \in X$.

We first show that $S$ is of type $\left(S_{+}\right)$, i.e., for any $\left\{u_{n}\right\} \subset X, u_{n} \rightarrow u_{0} \in X$ with $\lim \sup _{n \rightarrow \infty}\left(S u_{n}, u_{n}-u_{0}\right) \leq 0$, we have $u_{n} \rightarrow u_{0}$.

Since $T$ is linear and compact, $u_{n} \rightarrow u_{0}$ implies $T u_{n} \rightarrow T u_{0}$. It is easy to see that $\left(T u_{n}, u_{n}-u_{0}\right) \rightarrow\left(T u_{0}, u_{0}-u_{0}\right)=0$. Since $C$ is quasimonotone, $\lim \sup _{n \rightarrow \infty}\left(C u_{n}, u_{n}-u_{0}\right) \geq 0$. If $\limsup _{n \rightarrow \infty}\left(S u_{n}, u_{n}-u_{0}\right) \leq 0$, we have $\lim \sup _{n \rightarrow \infty}\left(A u_{n}, u_{n}-u_{0}\right) \leq 0$.

Assume that (3) is true, and let $m>0$ be the strong monotonicity constant of $A$. Then we have

$$
\begin{gathered}
m\left\|u_{n}-u_{0}\right\|^{2} \leq\left(A u_{n}-A u_{0}, u_{n}-u_{0}\right) \leq\left(A u_{n}, u_{n}-u_{0}\right)-\left(A u_{0}, u_{n}-u_{0}\right), \\
\underset{n \rightarrow \infty}{\limsup } m\left\|u_{n}-u_{0}\right\|^{2} \leq \limsup _{n \rightarrow \infty}\left(A u_{n}, u_{n}-u_{0}\right)-\lim _{n \rightarrow \infty}\left(A u_{0}, u_{n}-u_{0}\right) \leq 0 .
\end{gathered}
$$

Thus, $u_{n} \rightarrow u_{0}$.

If (4) is true, $A$ is monotone and of type $(S)$. Thus,

$$
\begin{gathered}
\left(A u_{n}, u_{n}-u_{0}\right)=\left(A u_{n}-A u_{0}, u_{n}-u_{0}\right)+\left(A u_{0}, u_{n}-u_{0}\right) \geq\left(A u_{0}, u_{n}-u_{0}\right), \\
\liminf _{n \rightarrow \infty}\left(A u_{n}, u_{n}-u_{0}\right) \geq \liminf _{n \rightarrow \infty}\left(A u_{0}, u_{n}-u_{0}\right)=0 .
\end{gathered}
$$

This says that if $\limsup _{n \rightarrow \infty}\left(A u_{n}, u_{n}-u_{0}\right) \leq 0$ then $\lim _{n \rightarrow \infty}\left(A u_{n}, u_{n}-u_{0}\right)=$ 0 , which, by the assumption that $A$ is of type $(S)$, implies $u_{n} \rightarrow u_{0}$. Hence, $S: X \rightarrow X^{*}$ is of type $\left(S_{+}\right)$.

By the degree theory for operators of type $\left(S_{+}\right)$(see Browder [2]), in order to show that $A u-T u+C u=f$ or $S u=0$ is solvable, we only need to show that there exists $b>0$ such that $S u+t J u \neq 0$, for any $u \in \partial B(0, b)$ and $t>0$ (see Guan [6]). Equivalently, we only need to show that the solutions of $S u+t J u=0$ are uniformly bounded with respect to $t \in(0, \infty)$.

If this is not true, there exists $\left\{u_{n}\right\} \subset X,\left\{t_{n}\right\} \subset(0, \infty)$, such that $A u_{n}-$ $T u_{n}+C u_{n}-f+t_{n} J u_{n}=0$ and $\left\|u_{n}\right\| \rightarrow \infty$.

Now we are going to show that there exists $\varepsilon>0$ such that $\left(C u_{n}, u_{n}\right) \geq$ $\varepsilon\left\|u_{n}\right\|^{p+1}$ for all $n$.

If this is not true, there exists a subsequence of $\left\{u_{n}\right\}$, again called $\left\{u_{n}\right\}$, such that $\left(C u_{n} /\left\|u_{n}\right\|, u_{n} /\left\|u_{n}\right\|\right) \rightarrow 0$. Since $\left\|u_{n} /\right\| u_{n}\|\|=1$ and $X$ is reflexive, we may assume that $u_{n} /\left\|u_{n}\right\| \rightarrow u_{0}$ for some $u_{0} \in X$.

We have

$$
\left(t_{n} J u_{n}, u_{n}\right)+\left(A u_{n}, u_{n}\right)-\left(T u_{n}, u_{n}\right)+\left(C u_{n}, u_{n}\right)-\left(f, u_{n}\right)=0,
$$


from which it follows that

$$
t_{n}\left\|u_{n}\right\|^{2} \leq\left(T u_{n}, u_{n}\right)+\left(f, u_{n}\right), \quad t_{n} \leq\|T\|+\left|\left(f, \frac{u_{n}}{\left\|u_{n}\right\|}\right) \frac{1}{\left\|u_{n}\right\|}\right| .
$$

Thus, $\left\{t_{n}\right\}$ is bounded. Now, $C u_{n}=-A u_{n}+T u_{n}-t_{n} J u_{n}+f$ and

$$
\begin{aligned}
C \frac{u_{n}}{\left\|u_{n}\right\|} & =\frac{1}{\left\|u_{n}\right\|^{p}}\left(-A u_{n}+T u_{n}-t_{n} J u_{n}+f\right) \\
& =-\frac{A u_{n}}{\left\|u_{n}\right\|^{p}}+T\left(\frac{u_{n}}{\left\|u_{n}\right\|^{p}}\right)-t_{n} J\left(\frac{u_{n}}{\left\|u_{n}\right\|^{p}}\right)+\frac{f}{\left\|u_{n}\right\|^{p}} .
\end{aligned}
$$

Since $\left\|u_{n}\right\| \rightarrow \infty, p>1$, it is easy to see that $T\left(u_{n} /\left\|u_{n}\right\|^{p}\right) \rightarrow 0, t_{n} J\left(u_{n} /\left\|u_{n}\right\|^{p}\right)$ $\rightarrow 0$, and $f /\left\|u_{n}\right\|^{p} \rightarrow 0$. Let $s$ be any number in $(q, p)$, by assumption (3) or (4). We have

$$
\frac{A u_{n}}{\left\|u_{n}\right\|^{p}}=\frac{1}{\left\|u_{n}\right\|^{p-s}} \frac{A u_{n}}{\left\|u_{n}\right\|^{s}}=\frac{1}{\left\|u_{n}\right\|^{p-s}} A \frac{u_{n}}{\left\|u_{n}\right\|^{s / q}} .
$$

Since $s>q,\left\|u_{n}\right\| \rightarrow \infty, u_{n} /\left\|u_{n}\right\|^{s / q} \rightarrow 0$. Since $A$ is demicontinuous, we have $A u_{n} /\left\|u_{n}\right\|^{s / q} \rightarrow 0$ as $n \rightarrow \infty$. Since $\left\|u_{n}\right\|^{p-s} \rightarrow \infty$, we have $A u_{n} /\left\|u_{n}\right\|^{p} \rightarrow 0$. Hence, $C u_{n} /\left\|u_{n}\right\| \rightarrow 0$, and we can easily show that

$$
\lim _{n \rightarrow \infty}\left(C u_{n} /\left\|u_{n}\right\|, u_{n} /\left\|u_{n}\right\|-u_{0}\right)=0 .
$$

Since $C$ is of type $(M)$, the conditions $u_{n} /\left\|u_{n}\right\|-u_{0}, C u_{n}\left\|u_{n}\right\| \rightarrow 0$, and $\lim _{n \rightarrow \infty}\left(C u_{n} /\left\|u_{n}\right\|, u_{n} /\left\|u_{n}\right\|-u_{0}\right)=0$ imply $C u_{0}=0$. By our assumption on $C$, we have $u_{0}=0$; so $u_{n} /\left\|u_{n}\right\| \rightarrow 0$.

Now, if (3) is true, we have

$$
T u_{n}=A u_{n}+C u_{n}+t_{n} J u_{n}-f, \quad\left(T u_{n}, u_{n}\right) \geq\left(A u_{n}, u_{n}\right)-\left(f, u_{n}\right),
$$

and

$$
\begin{aligned}
\limsup _{n \rightarrow \infty}\left(T \frac{u_{n}}{\left\|u_{n}\right\|}, \frac{u_{n}}{\left\|u_{n}\right\|}\right) & \geq \limsup _{n \rightarrow \infty}\left[\frac{1}{\left\|u_{n}\right\|^{2}}\left(A u_{n}, u_{n}\right)-\frac{1}{\left\|u_{n}\right\|}\left(f, \frac{u_{n}}{\left\|u_{n}\right\|}\right)\right] \\
& \geq \limsup _{n \rightarrow \infty} \frac{1}{\left\|u_{n}\right\|^{2}} m\left\|u_{n}\right\|^{2}=m>0,
\end{aligned}
$$

where $m$ is the strong monotonicity constant of $A$. Since $T$ is completely continuous and $u_{n} /\left\|u_{n}\right\| \rightarrow 0$, we have $\left(T u_{n} /\left\|u_{n}\right\|, u_{n} /\left\|u_{n}\right\|\right) \rightarrow 0$. This is a contradiction.

If (4) is true, then

$$
\begin{gathered}
A u_{n}-T u_{n}+C u_{n}+t_{n} J u_{n}-f=0, \\
\left(A u_{n}, u_{n}\right)-\left(T u_{n}, u_{n}\right)+\left(C u_{n}, u_{n}\right)=-t_{n}\left(J u_{n}, u_{n}\right)+\left(f, u_{n}\right) \leq\left(f, u_{n}\right),
\end{gathered}
$$

and

$$
\begin{aligned}
& \frac{1}{\left\|u_{n}\right\|}\left(A u_{n}, \frac{u_{n}}{\left\|u_{n}\right\|}\right)-\left(T \frac{u_{n}}{\left\|u_{n}\right\|}, \frac{u_{n}}{\left\|u_{n}\right\|}\right)+\frac{1}{\left\|u_{n}\right\|}\left(C u_{n}, \frac{u_{n}}{\left\|u_{n}\right\|}\right) \\
& \quad \leq \frac{1}{\left\|u_{n}\right\|}\left(f, \frac{u_{n}}{\left\|u_{n}\right\|}\right) .
\end{aligned}
$$

Since $A(r u)=r^{q} A(u)$ for any $r \geq 0$ and $\left\|u_{n}\right\| \geq 1$, we have

$$
\left(A \frac{u_{n}}{\left\|u_{n}\right\|}, \frac{u_{n}}{\left\|u_{n}\right\|}\right)=\frac{1}{\left\|u_{n}\right\|^{q}}\left(A u_{n}, \frac{u_{n}}{\left\|u_{n}\right\|}\right) \leq \frac{1}{\left\|u_{n}\right\|}\left(A u_{n}, \frac{u_{n}}{\left\|u_{n}\right\|}\right) .
$$


Since $C(r u)=r^{p} C(u)$ for any $r \geq 0$ and $\left\|u_{n}\right\| \geq 1$, we have

Hence,

$$
\left(C \frac{u_{n}}{\left\|u_{n}\right\|}, \frac{u_{n}}{\left\|u_{n}\right\|}\right)=\frac{1}{\left\|u_{n}\right\|^{p}}\left(C u_{n}, \frac{u_{n}}{\left\|u_{n}\right\|}\right) \leq \frac{1}{\left\|u_{n}\right\|}\left(C u_{n}, \frac{u_{n}}{\left\|u_{n}\right\|}\right) \text {. }
$$

$$
\left(A \frac{u_{n}}{\left\|u_{n}\right\|}, \frac{u_{n}}{\left\|u_{n}\right\|}\right)-\left(T \frac{u_{n}}{\left\|u_{n}\right\|}, \frac{u_{n}}{\left\|u_{n}\right\|}\right)+\left(C \frac{u_{n}}{\left\|u_{n}\right\|}, \frac{u_{n}}{\left\|u_{n}\right\|}\right) \leq \frac{1}{\left\|u_{n}\right\|}\left(f, \frac{u_{n}}{\left\|u_{n}\right\|}\right) .
$$

Thus, $\lim \sup _{n \rightarrow \infty}\left((A-T+C) u_{n} /\left\|u_{n}\right\|, u_{n} /\left\|u_{n}\right\|\right) \leq 0$. As we did at the beginning of the proof, we can show that $A-T+C$ is of type $\left(S_{+}\right)$. This implies $u_{n} /\left\|u_{n}\right\| \rightarrow 0$, which is a contradiction.

Hence, we have $\left(C u_{n}, u_{n}\right) \geq \varepsilon\left\|u_{n}\right\|^{p+1}$ for some $\varepsilon>0$.

Now, $A u_{n}-T u_{n}+C u_{n}-f+t_{n} J u_{n}=0$ implies

$$
\begin{aligned}
0 & =\left(A u_{n}, u_{n}\right)-\left(T u_{n}, u_{n}\right)+\left(C u_{n}, u_{n}\right)-\left(f, u_{n}\right)+t_{n}\left(J u_{n}, u_{n}\right) \\
& \geq-\|T\|\left\|u_{n}\right\|^{2}+\varepsilon\left\|u_{n}\right\|^{p+1}-\|f\|\left\|u_{n}\right\| \rightarrow \infty,
\end{aligned}
$$

since $p>1$ and $\left\|u_{n}\right\| \rightarrow \infty$. This is a contradiction.

So we have that the solutions of $S u+t J u=0$ are uniformly bounded for any $t \in[0, \infty)$.

Remark. Theorem 3 is valid under more general conditions on $C$ as follows: $C=C_{1}+C_{2}: X \rightarrow X^{*}$ is demicontinuous and quasimonotone, $C_{1}$ is of type $(M)$, and there exists $p>1$ such that: $C_{1}(r u)=r^{p} C_{1}(u)$ for any $r>0$ and $u \in X,\left(C_{1} u, u\right) \geq 0$, and $C_{1}(u)=0$ implies $u=0$. Moreover, $\left(C_{2} u, u\right) \geq 0$, and there are positive constants $a, b$ and $p_{0}<p$ such that $\left\|C_{2} u\right\| \leq a\|u\|^{p_{0}}+b$ for all $u \in X$ with large $\|u\|$.

It should be pointed out that if $C$ is generalized pseudomonotone, then $C$ is of type $(M)$ and quasimonotone. If $C$ is completely continuous, then $C$ is generalized pseudomonotone.

\section{EXAMPLES}

The Von Kármán Equations

$$
\begin{cases}\Delta^{2} v=-[u, u] & \text { in } \Omega, \\ \Delta^{2} u=-\lambda \Delta u+[v, u]+f & \text { in } \Omega, \\ u=\frac{\partial u}{\partial \gamma}=v=\frac{\partial v}{\partial \gamma}=0 & \text { on } \partial \Omega\end{cases}
$$

can be put in the form of a nonlinear operator equation, $n-\lambda T u+C u=f$, with $u \in X=W_{0}^{2,2}(\Omega)$, and operators $T$ and $C$ satisfy the conditions of our results. These equations represent a two-dimensional model of the buckling of a thin elastic plate where the middle surface occupies a region $\Omega \subset R^{2}, u$ is the vertical displacement, and $v$ is the Airy stress function. For details, see Ciarlet and Rabier [5].

The following example of a monotone operator which satisfies $A(r u)=$ $r^{q} A(u)$, for any $r>0$ and some $q>1$, is given by Alikakos and Rostamain in [1]: Let $\Omega$ be a smoothly bounded domain in $R^{n}, n \geq 1, X=L^{m+1}(\Omega)$, $m>1$, and define the operator $A: X \rightarrow X^{*}$ by

$$
(A u, v)=\int_{\Omega}|u|^{m-1} u v d x, \quad u, v \in X=L^{m+1}(\Omega) .
$$

For more examples, the reader is referred to Kesavan [8] and Milojevic $[10,11]$. 


\section{REFERENCES}

1. N. Alikakos and R. Rostamain, Lower bound estimates and separable solutions for homogeneous equations of evolution in Banach space, J. Differential Equations 43 (1982), 323-344.

2. F. E. Browder, Fixed point theory and nonlinear problems, Bull. Amer. Math. Soc. (N.S.) 9 (1983), 1-39.

3. F. E. Browder and B. A. Ton, Nonlinear functional equations in Banach space and elliptic super-regularization, Math. Z. 106 (1968) 1-16.

4. Y. Chen, Solvability of nonlinear perturbations of linear operator equations with parameters, Appl. Anal. 44 (1992), 209-222.

5. P. G. Ciarlet and P. Rabier, Les equations de Von Kármán, Lecture Notes in Math., vol. 826, Springer-Verlag, Berlin, 1980.

6. Z. Guan, On operators of monotone type in Banach space, Ph.D. Dissertation, Univ. South Florida, Tampa, FL, 1990.

7. A. G. Kartsatos and R. Mabry, On the solvability in Hilbert space of certain nonlinear operator equations depending on parameters, J. Math. Anal. Appl. 120 (1986), 670-678.

8. S. Kesavan, Existence of solution by the Galerkin method for a class of nonlinear problem, Appl. Anal. 16 (1983), 279-290.

9. N. G. Lloyd, Degree theory, Cambridge Univ. Press, London and New York, 1978.

10. P. S. Milojević, Solvability of some semilinear equations with strong nonlinearities and application to elliptic problems, Appl. Anal. 25 (3) (1987), 181-196.

11. __ Solvability of semilinear equations with strong nonlinearities and applications to elliptic boundary value problems, Comment. Math. Univ. Carolin. 28 (1987), 735-750.

12. D. Pascali and J. Sburlan, Nonlinear mappings of monotone type, Sijthoff and Noordhoof, Bucharest, 1978.

Department of Mathematics, University of Wisconsin-Eau Claire, Eau Claire, WisCONSIN 54702

E-mail address: GUANZQUWEC.EDU 